\title{
Shakhbazian compact galaxy groups
}

\section{Photometric and spectroscopic study of ShCG 8, ShCG 14, ShCG 19, ShCG 22*}

\author{
H. Tovmassian ${ }^{1}$, H. Tiersch ${ }^{2}$, V. H. Chavushyan ${ }^{1}$, G. H. Tovmassian ${ }^{3}$, \\ S. G. Navarro ${ }^{4}$, S. Neizvestny ${ }^{5}$, and J. P. Torres-Papaqui ${ }^{1}$ \\ ${ }^{1}$ Instituto Nacional de Astrofísica Optica y Electrónica, AP 51 y 216, CP 72000, Puebla, Pue., Mexico \\ e-mail: [hrant; vahram; papaqui]@inaoep.mx \\ 2 Sternwarte Königsleiten, 81477 München, Leimbachstr. 1a, Germany \\ e-mail: htiersch@uni.de \\ 3 OAN, UNAM, Ensenada, BC, Mexico \\ e-mail: gag@astrosen.unam.mx \\ ${ }^{4}$ IAC, Tenerife, Spain \\ e-mail: silvana@ll.iac.es \\ 5 Special Astrophysical Observatory, RAS, Nizhny Arkhyz, Russia \\ e-mail: neiz@sao.ru
}

Received 22 September 2004 / Accepted 21 April 2005

\begin{abstract}
We present the results of a detailed spectroscopic and photometric study of four Shakhbazian compact groups: ShCG 8, ShCG 14, ShCG 19, and ShCG 22. We determined the redshifts of member galaxies and radial velocity dispersions of groups. We also studied the distribution of the surface brightness of member galaxies in $R$ and determined their morphological types. The profiles of the surface brightness versus semi-major axis, the curves of isophotal twisting, and Fourier parameter $a_{4}$ are constructed. We found that some members of groups are in the process of interaction. We determined physical parameters of groups: virial masses, luminosities, mass-to-luminosity ratios, and the crossing times. On the basis of the results obtained in this and our previous works, the general properties of 22 ShCGs are discussed.
\end{abstract}

Key words. galaxies: clusters: general - galaxies: distances and redshifts - galaxies: interactions - galaxies: general galaxies: kinematics and dynamics - galaxies: photometry

\section{Introduction}

In 1995 we commenced a spectroscopic and detailed photometric study of Shakhbazian compact groups (ShCGs). The list of ShCGs contains 377 groups (Shakhbazian 1973; Shakhbazian \& Petrosian 1974; Petrosian 1974, 1978; Baier et al. 1974; Baier \& Tiersch 1975, 1976a, 1976b, 1978, 1979). ShCGs were found by an eye inspection of the POSS prints. They were selected as dense groups of mainly red galaxies, the images of many of which were often hard to distinguish from those of stars. Through follow-up spectral observations (Tiersch et al. 2002, hereafter Paper I; Tovmassian et al. 2003a, hereafter Paper II, 2003b, hereafter Paper III, 2004a, hereafter Paper IV, 2004b, hereafter Paper V) it was found that some objects in the area of groups which were assumed in original papers to be stars, are in reality galaxies. On the other hand, only a

^ Figures 1 and 3-10 are only available in electronic form at http://www . edpsciences.org few assumed members of ShCGs turned out to be stars. Hence, the great majority of the assumed members of these groups are galaxies.

Many ShCGs, like ShCG 19 and ShCG 38 (Fig. 1), are very dense formations. Probably due to the compactness of images of the member galaxies, which on the POSS prints are almost indistinguishable from those of stars, these two groups and also some others were missed in other compact group lists, e.g. HCG list (1982). ShCGs generally consist of up to 15 members, while the distances between member galaxies are typically 3-5 times their diameters. The space density of many of ShCGs reaches about $10^{4}-10^{5}$ galaxies per $\mathrm{Mpc}^{3}$. The groups have a "cigar"-like, prolate spheroid configuration (Oleak et al. 1995). Numerical $N$-body simulations have showen that compact groups should undergo fast dynamical evolution, which will result in coalescence of member galaxies to one giant elliptical galaxy (Barnes 1985, 1989; Mamon 1986; Bode et al. 1993). It means that processes of dynamical 
Table 1. Positions of groups ShCG 8, ShCG 14, ShCG 19, ShCG 22, and Galactic extinction in $R$.

\begin{tabular}{rccc}
\hline \hline ShCG & $\begin{array}{c}\text { RA } \\
(2000)\end{array}$ & $\begin{array}{c}\text { Dec } \\
(2000)\end{array}$ & $Q_{R}$ \\
\hline 8 & $16^{\mathrm{h}} 03^{\mathrm{m}} 41^{\mathrm{s}}$ & $52^{\circ} 21^{\prime} 09^{\prime \prime}$ & $0^{\mathrm{m}} 042$ \\
14 & $14^{\mathrm{h}} 25^{\mathrm{m}} 20^{\mathrm{s}}$ & $47^{\circ} 15^{\prime} 00^{\prime \prime}$ & $0 \cdot 043$ \\
19 & $13^{\mathrm{h}} 28^{\mathrm{m}} 30^{\mathrm{s}}$ & $15^{\circ} 50^{\prime} 20^{\prime \prime}$ & 0.074 \\
22 & $15^{\mathrm{h}} 45^{\mathrm{m}} 41^{\mathrm{s}}$ & $55^{\circ} 07^{\prime} 18^{\prime \prime}$ & $0 \cdot 040$ \\
\hline
\end{tabular}

friction, tidal interaction between galaxies, and galaxy merging may be observed in ShCGs.

The large sample of ShCGs implies that ShCGs may be at different stages of dynamical and morphological evolution. Therefore, the study of a large number of ShCGs may be very helpful for understanding the evolution of compact groups.

The aim of our program is collection of a large number of data on ShCGs. The results of the study of 18 ShCGs are presented Papers 1-5. We have obtained spectra of many dozens of galaxies in these groups, and measured their redshifts, which allowed us to confirm that most of them are accordant redshift members of corresponding groups. We also obtained the images of these groups in $B V R$, measured the magnitudes of member galaxies in the corresponding bands, and determined their morphological types. We studied the brightness distribution of member galaxies and showed that some of them are in the process of interaction. In this paper we present the results of the study of four groups: ShCG 8, ShCG 14, ShCG 19, ShCG 22.

\section{Observations and results}

The coordinates of the centers of the studied groups given in Table 1 are taken from Stoll et al. (1996). In the last column of Table 1 the Galactic extinction in $R$ (taken from the NED, Schlegel et al. 1998) is given.

\subsection{Spectroscopy}

Spectroscopic observations of 23 objects in the fields of ShCG 8, ShCG 14, ShCG 19, and ShCG 22 were carried out with the $2.1 \mathrm{~m}$ telescope of the Guillermo Haro Observatory in Cananea, Mexico, operated by the National Institute of Astrophysics, Optics and Electronics. Spectra were obtained during several observing runs in April 1996; May and July 1997, May and August 1998; March 1999, and February 2004. The Faint Object Spectrograph and Camera (Zickgraf et al. 1997) was used. Most of the observations were done with mean dispersion $5.5 \AA / p x l$ and mean resolution $13 \AA$. In a few cases the dispersion $8.2 \AA / \mathrm{pxl}$ and the resolution $18 \AA$ were applied.

Redshifts were measured using the IRAF and MIDAS programs. The sky lines were removed by a conventional program. Absorption lines of $\mathrm{H} \alpha, \mathrm{MgIb}, \mathrm{Fe}$, and $\mathrm{NaD}$ were identified in the spectra of almost all galaxies. In the spectra of some galaxies the $\mathrm{HK}$ absorption lines and $G$ bands were also identified. Emission lines $\mathrm{H}_{\alpha}$ or $\mathrm{H}_{\beta}$ were observed in the spectra of a few galaxies. In the spectra of ShCG 14-2 some forbidden emission lines were also observed. The spectra of emission line galaxies is presented in Fig. 2. According to the diagnostic diagram (Veilleus \& Osterbrock 1987) galaxy ShCG 14-2 may be a starburst or NELG. Other emission-line galaxies are not possible to classify due to the presence of only one or two emission lines. For measurements of redshifts the profile of each observed line was fitted by a Gaussian. The redshift of a galaxy was determined as the mean of measurements of individual lines. The dispersion of redshifts of individual lines determines the error. The radial velocities (RV) of galaxies were generally measured with an accuracy of about $200 \mathrm{~km} \mathrm{~s}^{-1}$. In the case of very faint galaxies the uncertainties may reach $\approx 400 \mathrm{~km} \mathrm{~s}^{-1}$. The measured RVs are presented in Table 2. Four galaxies with emission lines are marked by "e". RVs have been corrected for solar motion according to $\Delta v=300 \sin l \cdot \cos b \mathrm{~km} \mathrm{~s}^{-1}$. We found that one of the supposed members in ShCG 8 (No. 7) and object No. 3 in ShCG 19 are stars. Galaxy No. 3 with emissionline spectra in ShCG 8 is a foreground one projected over the group.

\subsection{Direct imaging and photometry}

Photometric observations in $R$ band were done with the $1.5 \mathrm{~m}$ telescope of the National Astronomical Observatory in San Pedro Mártir, Mexico, in March and December 1998 and in March and April 1999. During observations the seeing was better than $2^{\prime \prime}$. The TEK CCD detector used in observations has a $1024 \times 1024$ array of $24 \times 24 \mu \mathrm{m}$ pixels. The obtained images were corrected for flat fields using the twilight images of blank sky areas (Christian et al. 1985).

The images of the four studied groups are presented in the left panels of Figs. 3-6, in the right panels of which the isophotes of the observed galaxies are presented. The latter are chosen arbitrarily to reveal possible signs of interaction between member galaxies. The units of isophotes for each group are given in corresponding figure captions, where the galaxy identification numbers are from Stoll et al. (1996). Magnitudes are calibrated in the Kron-Cousins photometric system, and the star cluster NGC 4147 served as a standard. Reduction of images was done with the MIDAS program. In the case of isolated images of galaxies, a limit of the surface brightness generally lower than $\mu=26.5 / \operatorname{arcsec}^{2}$ is reached. The magnitudes are estimated within this contour. The diameters $D$ of galaxies and the axial ratios $b / a$ are determined by the same contour. The overlapping halos of images of galaxies were separated by extrapolation of the fitted ellipses in the not overlapped part down to the mentioned limiting surface brightness. The measured magnitudes are corrected for Galactic extinction $Q_{R}$ within our Galaxy (Col. 4 of Table 1). The observed groups are relatively nearby, therefore the $\mathrm{K}$ correction is neglected. The estimated accuracy of magnitudes of isolated images is about 0.06 , but the error may be higher in the case of overlapping halos.

If spiral structure is not apparent on the images of galaxies, the morphological types were determined by means of the profiles surface brightness, $\mu$, versus the semi-major axis $a$, 

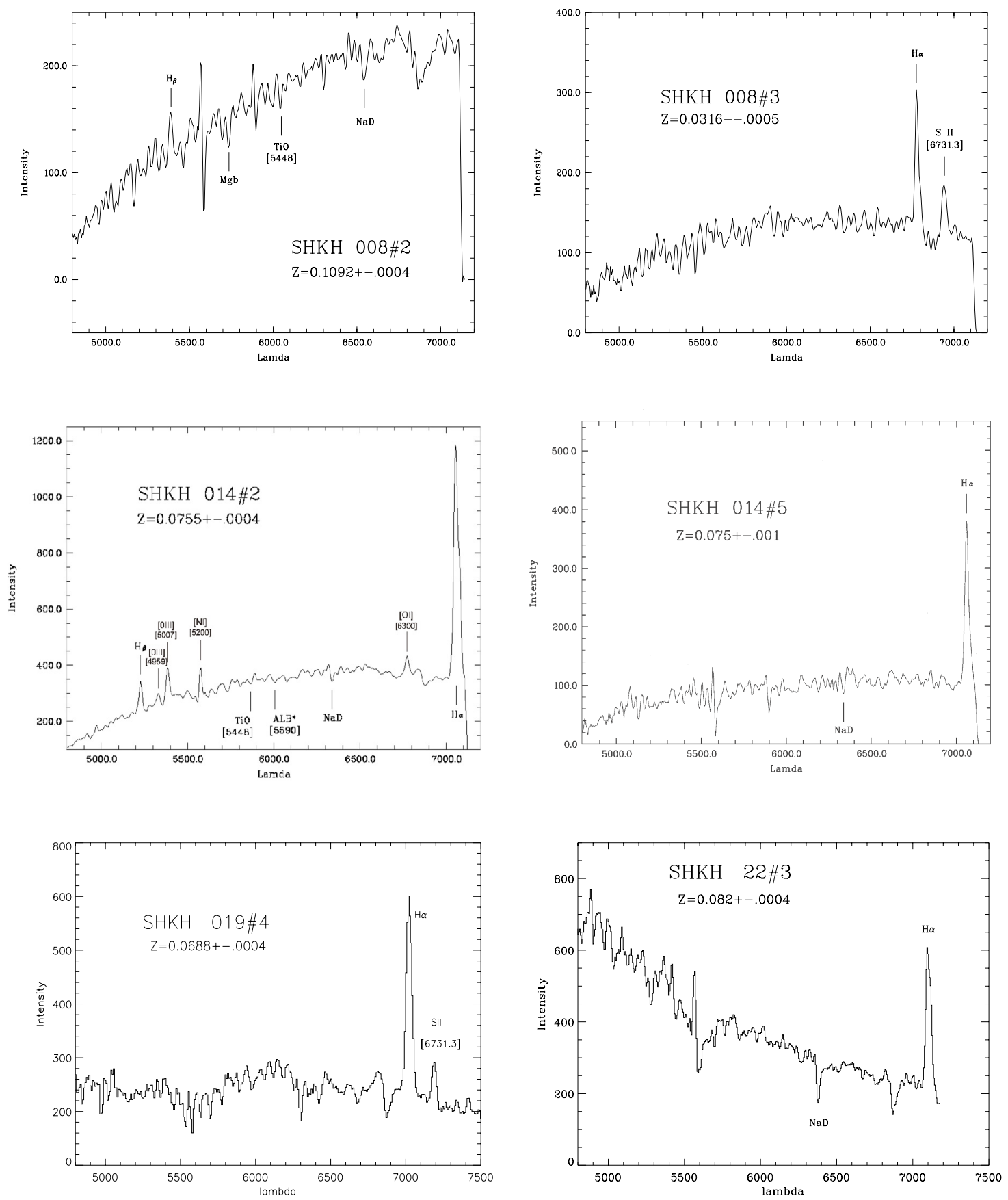

Fig. 2. The spectra of galaxies ShCG 8-2, ShCG 8-3 (foreground), ShCG 14-2, ShCG 14-5, ShCG 19-4, and ShCG 22-3 with H $\alpha$ and/or $\mathrm{H} \beta$ emission lines.

or $a^{1 / 4}$. Elliptical galaxies have a straight profile on the $\mu-$ $a^{1 / 4}$ graph (de Vaucouleurs 1948). However, all $\mu-a^{1 / 4}$ profiles of the galaxies studied in this paper significantly deviate from a straight line; hence, we present only the $\mu-a$ profiles. In the case of S0 and spiral galaxies the $\mu-a$ curve may be decomposed into the bulge and disc components (Kent 1985; Schombert \& Bothun 1987). The bulge in a spiral galaxy is less dominant and its profile steeper than that of a lenticular galaxy (Kent 1985). Thus, depending on the relative size of the bulge and the steepness of its profile, the galaxy was classified as S0 or S type. Kent (1985) mentioned, however, that not all galaxies can be fitted by these simple functions. Deviations from ellipticity in both the core and the envelope, as well as deviations from concentric ellipses, are sometimes observed (Pildis et al. 1995). Such deviations may indicate that a galaxy has undergone an interaction, though this may not be true in every case.

We constructed the curves of isophotal twisting of galaxies PA $-a$, where PA is the position angle. These curves may indicate the presence of mutual tidal perturbations or galaxy collisions (Kormendy 1982; di Tullio 1979). In order to characterize the properties of individual galaxies from the surface photometry, we also calculated the Fourier parameter $a_{4}$, which describes deviations from perfect elliptical isophotes. 
Table 2. Radial velocities of member galaxies in groups ShCG 8, ShCG 14, ShCG 19, and ShCG 22.

\begin{tabular}{ll|ll|ll|ll}
\hline \hline \multicolumn{2}{c|}{ ShCG 8 } & \multicolumn{2}{|c|}{ ShCG 14 } & \multicolumn{2}{c|}{ ShCG 19 } & \multicolumn{2}{c}{ ShCG 22 } \\
$g$ & $\begin{array}{l}v_{\text {rad }} \\
{\left[\mathrm{km} \mathrm{s}^{-1}\right]}\end{array}$ & $g$ & $\begin{array}{l}v_{\text {rad }} \\
{\left[\mathrm{km} \mathrm{s}^{-1}\right]}\end{array}$ & $g$ & $\begin{array}{l}v_{\text {rad }} \\
{\left[\mathrm{km} \mathrm{s}^{-1}\right]}\end{array}$ & $g$ & $\begin{array}{l}v_{\text {rad }} \\
{\left[\mathrm{km} \mathrm{s}^{-1}\right]}\end{array}$ \\
\hline 1 & 33200 & 1 & 21710 & 1 & 20020 & 1 & 24590 \\
2 & $33400 \mathrm{e}$ & 2 & $22730 \mathrm{e}$ & 2 & 21040 & 2 & 24620 \\
3 & $10200 \mathrm{e}$ & 3 & 21770 & 3 & star & 3 & $24620 \mathrm{e}$ \\
4 & 32800 & 4 & 21950 & 4 & $21040 \mathrm{e}$ & 4 & 25100 \\
5 & 32800 & 5 & $22850 \mathrm{e}$ & 5 & 20380 & & \\
6 & 33000 & 6 & 22790 & & & & \\
7 & star & 7 & 22520 & & & & \\
\hline
\end{tabular}

According to Bender (1988), elliptical galaxies may be separated into two types - with "box-like" isophotes $\left(a_{4}<0\right)$ and "disk-like" isophotes $\left(a_{4}>0\right)$. Bender \& Möllenhoff (1987) mentioned that the boxiness most likely originates from merging or tidal disruptions of companion galaxies. Though according to Lima Neto \& Combes (1995), Bettoni \& Fasano (1993, 1995), and Fasano \& Bettoni (1994), they are not strictly correlated with interaction and merging processes. The profiles $\mu-a$, and also the profiles PA $-a$ and $a_{4}-a$ for galaxies in ShCG 8, ShCG 14, ShCG 19, ShCG 22, are presented in Figs. 7-10, respectively. The last figures show that no one galaxy in the studied four ShCGs have "box-like" isophotes; i.e. all observed galaxies are "disk-like".

The results of the photometry of member galaxies in ShCG 8, ShCG 14, ShCG 19, ShCG 22 are presented in Table 3, in which the following information is given: Col. 1 - the galaxy identification number, Col. 2 - the magnitude in $R_{26.5}$, Col. 3 - the colour $R-K_{\text {total }}$ for galaxies observed by 2MASS (Jarrett et al. 2000, and http://www.ipac. caltech.edu/2mass), Col. 4 - the axial ratio $b / a$, Col. 5 - the diameter of the galaxy; Col. 6 - the morphological type.

\section{Discussion}

ShCG 8. According to the original list (Shakhbazian 1973; Stoll et al. 1996) the group consists of seven galaxies. Spectral observations revealed, however, that one of the supposed members of this group, No. 7, is a star. Galaxy No. 3 has smaller redshift and is projected over the group; hence, the group consists of 5 accordant redshift galaxies. The bridges between galaxies 1 and 2, and 6 and 1 show that these three galaxies are interacting. A faint infrared source IRAS Z16024+5229 is located at about $40^{\prime \prime}$ to the north-east of galaxies 1 and 2 and may be identified with one of them. Since galaxy No. 2 has emission line $(\mathrm{H} \beta)$, perhaps it is the FIR-emitter.

ShCG 14. Spectral observations showed that galaxy No. 7 located at about $1.5^{\prime}$ to the south of the group and not included in the original list (Shakhbazian 1973; Stoll et al. 1996) belongs to the group, so that the group consists of seven accordant redshift members. Though the group is a sufficiently dense one, the isophotes (Fig. 4) do not show any apparent
Table 3. Photometric parameters of galaxies in ShCG 8, ShCG 14, ShCG 19, ShCG 22.

\begin{tabular}{|c|c|c|c|c|c|}
\hline Gal & $R$ & $R-K_{\mathrm{t}}$ & $b / a$ & $D\left[{ }^{\prime \prime}\right]$ & type \\
\hline \multicolumn{6}{|c|}{ ShCG 8} \\
\hline 1 & 16.95 & 3.70 & 0.75 & 9.1 & S0 \\
\hline 2 & 17.44 & & 0.97 & 10.1 & S \\
\hline $3 *$ & 17.45 & & 0.75 & 14.3 & S \\
\hline 5 & 17.86 & & 0.58 & 9.8 & So \\
\hline 6 & 18.38 & & 0.77 & 7.4 & S \\
\hline \multicolumn{6}{|c|}{ ShCG 14} \\
\hline 1 & 15.86 & 2.82 & 0.95 & 17.2 & $\mathrm{E} / \mathrm{S} 0$ \\
\hline 2 & 16.42 & 2.80 & 0.72 & 15.8 & So \\
\hline 3 & 16.23 & 3.13 & 0.59 & 21.6 & $\mathrm{~S} 0 / \mathrm{S}$ \\
\hline 4 & 16.85 & 3.37 & 0.94 & 9.8 & So \\
\hline 5 & 18.12 & & 0.77 & 8.2 & $\mathrm{E} / \mathrm{S} 0$ \\
\hline 6 & 18.06 & & 0.76 & 8.4 & $\mathrm{E} / \mathrm{S} 0$ \\
\hline 7 & 17.33 & & 0.58 & 19.5 & S \\
\hline \multicolumn{6}{|c|}{ ShCG 19} \\
\hline 1 & 16.59 & 3.75 & 0.82 & 9.0 & So \\
\hline 2 & 16.46 & & 0.47 & 14.6 & $\mathrm{~S} 0 / \mathrm{S}$ \\
\hline 4 & 17.52 & & 0.78 & 9.8 & $\mathrm{~S} 0 / \mathrm{S}$ \\
\hline 5 & 17.64 & & 0.97 & 7.0 & So \\
\hline \multicolumn{6}{|c|}{ ShCG 22} \\
\hline 1 & 15.54 & 2.80 & 0.84 & 16.6 & So \\
\hline 2 & 16.12 & 2.62 & 0.73 & 17.6 & So \\
\hline 3 & 15.78 & 2.67 & 0.60 & 18.4 & S \\
\hline 4 & 15.78 & 2.56 & 0.94 & 21.7 & So \\
\hline 5 & 18.58 & & 0.52 & 9.5 & S0 \\
\hline
\end{tabular}

Foreground galaxy.

signs of interaction. However, since galaxy 2 is a starburst (or NELG), and galaxy 5 has an $\mathrm{H} \alpha$ in emission, we may suppose that starburst processes and emission lines could probably be triggered by interaction of these two galaxies with each other or with other members of the group. Furthermore, galaxy 2 is identified with the faint infrared source at the position $14^{\circ} 25^{\prime} 20.0^{\prime \prime}$ (Tovmassian et al. 1998). Another infrared source, IRAS F14234+4729, at $\approx 30^{\prime \prime}$ north-west from the center of the group may probably be associated with either member. These sources may be the result of interaction between the group galaxies.

ShCG 19. The cluster $\mathrm{ZwCl} 1326.4+1612$ with a size of about $18^{\prime}$ is located at about $8^{\prime}$ to the SW of the group center. The distance class of the cluster is $D$ (distant), and very probably members of the group ShCG 19 do not belong to the cluster. Our spectral observations showed that one of the supposed members of this group, object 3, is a star. Thus, ShCG 19 is a very dense group of four galaxies, and all four galaxies of the group seem to be embedded in a common envelope (Fig. 5). This is hardly a result of the photographic summation of the halos of individual galaxies, because the halos of galaxies are clearly enlarged. Therefore, we suppose that the group members may be interacting with each other. 
Table 4. Physical parameters of the studied ShCGs.

\begin{tabular}{lrrrr}
\hline \hline Parameter & ShCG & ShCG & ShCG & ShCG \\
& 8 & 14 & 19 & 22 \\
\hline$z$ & 0.1104 & 0.0730 & 0.0694 & 0.0825 \\
$a[\mathrm{kpc}]$ & 90 & 200 & 26 & 200 \\
$\mathrm{RVD}\left[\mathrm{km} \mathrm{s}^{-1}\right]$ & 190 & 560 & 235 & 230 \\
$R_{\mathrm{vir}}[\mathrm{kpc}]$ & 43 & 57 & 15 & 115 \\
$\mathcal{M}_{\mathrm{vir}}\left[10^{11} \mathcal{M}_{\odot}\right]$ & 17 & 196 & 10 & 65 \\
$L_{R}\left[10^{11} L_{\odot}\right]$ & 2.6 & 3.6 & 1.5 & 6.1 \\
$\mathcal{M} / L_{R}\left[\mathcal{M}_{\odot} / L_{\odot}\right]$ & 6 & 54 & 6 & 11 \\
$\tau_{\mathrm{c}}\left[10^{6}\right.$ years $]$ & 93 & 42 & 27 & 208 \\
\hline
\end{tabular}

ShCG 22. The center of the cluster $\mathrm{ZwCl} 1545.0+5515$, with a size of about $18^{\prime}$, is at $\approx 11^{\prime}$ to the SE from the group. However, the distance class of the cluster is ED; i.e. its radial velocity should be more than $60000 \mathrm{~km} \mathrm{~s}^{-1}$. It means that the group ShCG 22 is just projected over the cluster. We did not get the spectra of the faintest supposed member No. 5 of this group. However, direct image of the group (Fig. 6) shows that this dwarf galaxy seems to be in interaction with galaxy No. 4 which is identified with the infrared source IRAS Z15445+5515 (Tovmassian et al. 1998). Therefore, galaxy 5 very probably does belong to the group and is not a member of the distant cluster. The isophotes of galaxies (see Fig. 6) show that galaxies 2 and 3 are certainly interacting. Furthermore, galaxy 3 has an emission-line spectrum.

The physical parameters of groups. Using the results of the photometry of member galaxies in the observed groups and knowing their distances, we determined, as in other papers of this series (Papers 1-5), some important physical parameters of the groups presented in Table 4, in which the following information is given: line $1-$ the redshift $z$ (weighted by masses of member galaxies); line 2 - the length $a$ of the group $\left(H_{0}=55 \mathrm{~km} \mathrm{~s}^{-1} \mathrm{Mpc}^{-1}\right)$ determined by two most remote from each other members of the group; line 3 - the RVD, (weighted by the masses of galaxies); line 4 - the virial radius, $R_{\text {vir }}$, of the group (weighted by the masses of galaxies); line 5 - the virial mass, $\mathcal{M}_{\text {vir }}$; line 6 - the luminosity of the group, $L$, in solar units in $R$ band; line 7 - the mass-to-luminosity ratio, $\mathcal{M} / L$, in solar units; and line $8-$ the crossing time, $\tau_{\mathrm{c}}$. For details of determination of the above-mentioned parameters see Paper I.

The summary of the physical parameters of 22 ShCGs, determined in this paper and in Papers $\mathrm{I}-\mathrm{V}$, is presented below. We obtained spectra of 146 objects. Six of them turned out to be stars. Another seven objects are discordant redshift galaxies projected over the groups. As a result, $90 \%$ of the supposed member galaxies in corresponding groups are confirmed. Detailed photometric data was obtained for 181 galaxies, including seven discordant redshift ones.

The mean RVD of 22 studied groups is $\approx 330 \pm 154 \mathrm{~km} \mathrm{~s}^{-1}$. Meanwhile, the RVDs of rich galaxy clusters are higher, in the order of $\approx 1000 \mathrm{~km} \mathrm{~s}^{-1}$, (Zabludoff et al. 1990). The highest RVD, $640 \mathrm{~km} \mathrm{~s}^{-1}$ and $670 \mathrm{~km} \mathrm{~s}^{-1}$, are those of ShCG 360 and ShCG 361, respectively. The RVDs of ShCG 74W and ShCG 344 consisting of three and five members, respectively, are very small, $88 \mathrm{~km} \mathrm{~s}^{-1}$ and $115 \mathrm{~km} \mathrm{~s}^{-1}$, respectively. The virial masses of the studied groups are within $(10 \div 700) \times$ $10^{11} \mathcal{M}_{\odot}$. The derived mass of a group with a few members may, however, have some uncertainty for the unknown projection effect. The obtained values are typical of galaxy groups. The mean mass-to-luminosity ratio for 22 groups is $32 \pm 29$, and is higher than the dynamical mass-to-light ratio for ellipticals, $\approx 8$, (Karachentsev 1987). The highest mass-to-luminosity ratio, $\mathcal{M} / L=100$, belongs to ShCG 361 . The mean crossing time $\tau_{\mathrm{c}}$ of the $21 \mathrm{ShCGs},(127 \pm 87) \times 10^{6} \mathrm{yr}$. The crossing time of ShCG $74 \mathrm{~W}$ is very high, $\approx 600 \times 10^{6} \mathrm{yr}$. The recalculated mean crossing time of ShCGs is $\approx 70 \times 10^{6} \mathrm{yr}$ and is smaller than that of HCGs, $\approx 260 \times 10^{6} \mathrm{yr}$ (Hickson et al. 1992). One has to take into account that the real parameters of groups in both samples (HCGs and ShCGs) may in reality differ from the deduced values. It was shown that many HCGs are embedded in loose groups (Sulentic 1987; Rood \& Williams 1989; Rood \& Struble 1994). In a series of papers (Tovmassian et al. 2001; Tovmassian 2001, 2002; Tovmassian \& Chavushyan 2000; Tovmassian \& Tiersch 2001) it was shown that HCGs and ShCGs are the cores of larger, elongated groups, the members of which are gravitationally bound to the former.

Due to processes of interaction which may be frequent in the central areas of ShCGs, the spiral galaxies convert to S0/E types, as suggested by Toomre \& Toomre (1972), Barnes \& Hernquist (1992) and Mihos (1995). Detailed photometry showed that in almost every group there are interacting galaxies, explaining the high rate of galaxies of S0/E types: $77 \%$ (or $70 \%$, if all galaxies with uncertain type S/S0 are spiral galaxies). The loss of interstellar gas by galaxies caused by tidal forces and/or ram pressure may also explain the lack of spirals. The groups ShCG 8, ShCG 251, ShCG 344, and ShCG 376 are exceptions. About $84 \%$ of members in these groups are spiral galaxies. These groups may be in an early stage of formation. Elliptical galaxies in all studied ShCGs are very red, as the $B-V$ of many of them are about $1 . \mathrm{m} 0$ or more, similar to the reddest ellipticals (Buta et al. 1995). The mean $R-K_{\text {total }}$ colour of 23 members of the studied groups observed by 2 MASS is 2 . $91 \pm 0.55$.

FIR emission was detected in $\approx 7 \%$ of the ShCGs (Tovmassian et al. 1998), which is much less than in HCGs (64\%). The median redshift of extragalactic objects selected by their $60 \mu \mathrm{m}$ IRAS emission is $z=0.03$. (Kleinmann \& Kill 1987). This corresponds to a median FIR luminosity of $\approx 2 \times 10^{10} L_{\odot}$. Meanwhile, redshift $z$ of almost all of $\approx 70$ ShCGs studied spectroscopically are higher than 0.06 (Papers I-V, in preparation). Higher distances of ShCGs may partly explain the lack of many FIR and also the radio emitting galaxies in them (Tovmassian et al. 1998). Another reason for the absence of FIR and radio sources may be that ShCGs have a very elongated space configuration (Oleak et al. 1995), and are very probably in a quasi-stable state (Tovmassian 2002). In this case the members of a group have semi-regular movement in elongated orbits around its gravitational center. The rate of interaction and merging will be smaller than in the case of chaotic movement of member galaxies considered in $N$-body simulations. On the other hand, Klamer et al. (2004) 
mention that radio emission may not be triggered by galaxygalaxy interaction.

ShCGs were selected by an eye inspection of the POSS prints as dense groups consisting mainly of red galaxies, the images of many of which often were hard to distinguish from those of stars. It is noteworthy that no bright blue elliptical galaxies predicted by the environment-dominated models of evolution of galaxies (Zepf et al. 1991) were found in the studied groups.

\section{Conclusions}

We spectroscopically investigated 23 objects in the regions of four ShCGs - ShCG 8, ShCG 14, ShCG 19, and ShCG 22. We found that two of these objects are stars, and that one galaxy, ShCG 8-3, has discordant redshift. Twenty-one galaxies in the area of these four groups were observed in $R$. Important physical parameters of the studied groups are deduced (Table 4).

The results of the detailed study of ShCG 8, ShCG 14, ShCG 19, and ShCG 22 and of 18 other groups presented in Papers I-V are summarized below:

1. We determined RVs of 144 objects in 22 ShCGs. Six of the supposed members of groups turned out to be stars (objects ShCG 8-7, ShCG 19-3, ShCG 31-3, ShCG 38-5, ShCG 251-6, and ShCG 362-10). Four more stars (objects ShCG 188-11, ShCG 188-12, ShCG 362-7, and ShCG 362-8) were revealed by detailed photometry of 181 supposed members. Hence, the overwhelming majority of objects in the studied groups are, indeed, galaxies, although ShCGs have been selected by an eye search from the POSS prints as compact groups of compact galaxies. One could expect that some of these compact objects could in reality be stars.

2. RVs of galaxies in 22 groups prove that they are real physical entities and not a chance projection of field galaxies. Out of 144 spectroscopically observed members, only six or possibly seven (if we also include galaxy ShCG 376-6) are foreground galaxies.

3. The redshifts of the 22 ShCGs range from 0.0400 to 0.2180 , i.e.; they all are located well beyond the Local Supercluster.

4. The mass-weighted RVDs of the studied 22 ShCGs are between $90 \mathrm{~km} \mathrm{~s}^{-1}$ and $670 \mathrm{~km} \mathrm{~s}^{-1}$.

5. The virial radii are small enough and do not exceed $160 \mathrm{kpc}$.

6. The virial masses of the groups range from $\approx 10 \times 10^{11} \mathcal{M}_{\odot}$ to $\approx 720 \times 10^{11} \mathcal{M}_{\odot}$. These values are typical of galaxy groups and poor clusters. However, one has to take into account that the real masses may be higher since, as was shown by Tovmassian \& Tiersch (2001), ShCGs are the cores of larger, elongated systems.

7. The mass-to-luminosity ratio $\mathcal{M} / L_{R}$ of most of 22 ShCGs is within $3 \div 100$ with an average value $\mathcal{M} / L_{R}=32.0 \pm 29$.

8. The crossing time for 21 out of 22 ShCGs ranges from $30 \times$ $10^{6} \mathrm{yr}$ to $340 \times 10^{6} \mathrm{yr}$, which is much shorter than the Hubble age. One group, ShCG $74 \mathrm{~W}$, has higher $\tau_{\mathrm{c}}$ equal to $605 \times 10^{6} \mathrm{yr}$. The high value of $\tau_{\mathrm{c}}$ for this group may be due to the small RVD (90 $\left.\mathrm{km} \mathrm{s}^{-1}\right)$ deduced by using RVs of only three members.

9. The majority $(\approx 75 \%)$ of the galaxies in ShCGs are E and S0 galaxies. The loss of interstellar gas by galaxies caused by tidal forces and/or ram pressure of interaction between member galaxies may also play a role. Detailed photometry showed that in almost each group there are interacting galaxies.

10. Only a few galaxies in ShCGs have FIR and radio emission (Tovmassian et al. 1998, 1999), although according to $N$-body simulations it is expected that in dense environments of groups the number of interactions and merging will be high; and consequently there should be a high number of radio and FIR emitting galaxies. The absence of strong radio and FIR sources in compact groups may be explained by their large distances and by suggested semiregular oscillation of the group members in elongated orbits around its gravitational center (Tovmassian 2002).

Acknowledgements. We are grateful to the anonymous referee comments of whom improved the paper. H.M.T. acknowledges CONACYT for support through the Project 201-PY.44376/A-1, and the Königsleiten Observatory for partial financial support (accommodation) during July 2004. H.T. is grateful to the Government of the Land Brandenburg for the support of this work (Az 24-19/003; 2000) and to the DFG for grants TI 215/6-2, TI 215/6-3, MEX 111/1/98. H.T. and S.N. thank Mr. O. Beck for private financial support.

\section{References}

Baier, F. W., \& Tiersch, H. 1975, Astrofizika, 11, 221

Baier, F. W., \& Tiersch, H. 1976a, Astrofizika, 12, 7

Baier, F. W., \& Tiersch, H. 1976b, Astrofizika, 12, 409

Baier, F. W., \& Tiersch, H. 1978, Astrofizika, 14, 279

Baier, F. W., \& Tiersch, H. 1979, Astrofizika, 15, 33

Baier, F. W., Petrosian, M. B., Tiersch, H., \& Shakhbazian, R. K. 1974, Astrofizika, 10, 327

Barnes, J. E. 1985, MNRAS, 215, 517

Barnes, J. E. 1989, Nature, 338, 123

Barnes, J. E., \& Hernquist, L. 1992, Nature, 360, 715

Bender, R. 1988, A\&A, 193, L7

Bender, R., \& Möllenhoff, C. 1987, A\&A, 177, 71

Bettoni, D., \& Fasano, G. 1993, AJ, 105, 1291

Bettoni, D., \& Fasano, G. 1995, AJ, 109, 32

Bode, P. W., Cohn, N. H., \& Lugger, P. M. 1993, ApJ, 416, 17

Buta, B., Corvin, H. G. Jr., de Vaucouleurs, G., de Vaucouleurs, A., \& Longo, G. 1995, AJ, 109, 517

Christian, C. A., Adams, M., Barnes, J. V., et al. 1985, PASP, 97, 363

de Vaucouleurs, G. 1948, Ann. Ap., 11, 247

di Tullio, G. A. 1979, A\&AS, 37, 591

Fasano, G., \& Bettoni, D. 1994, AJ, 107, 1649

Hickson, P. 1982, ApJ, 255, 382

Hickson, P., Mendes de Oliveira, C., Huchra, J. P., \& Palumbo, G. G. C. 1992, ApJ, 399, 353

Jarrett, T. H., Chester, T., Cutri, R., et al. 2000, AJ, 119, 2498

Karachentsev, I. D. 1987, Dvojnye galaktiki (Moscow: Nauka), 76

Kent, S. M. 1985, ApJS, 59, 115

Klamer, K., Subrahmanyan, R., \& Hunstead, R. W. 2004, MNRAS, 351,101

Kleinmann, S. G., \& Kill, W. 1987, in Star Formation in Galaxies, ed. C. Persson, Washington DC (US Govt. Print. Off.), 559 
Kormendy, J. 1982, Morphology and Dynamics of Galaxies, ed. L. Mayor, \& M. Martinet, Geneva, 113

Lima Neto, G., \& Combes, F. 1995, A\&A, 294, 657

Mamon, G. A. 1986, ApJ, 307, 426

Mihos, J. C. 1995, ApJ, 438, L75

Oleak, H., Stoll, D., Tiersch, H., \& MacGillivray, H. T. 1995, AJ, 109, 1485

Peletier, R. F., Davies, R. L. Illingqworth, G. D., Davis, L. E., \& Cawson, M. 1990, AJ, 100, 1091

Petrosian, M. B. 1974, Astrofizika, 10, 471

Petrosian, M. B. 1978, Astrofizika, 14, 631

Pildis, R. A., Bregman, J. N., \& Schomberg, J. M. 1995, AJ, 110, 1498

Rood, H. J., \& Williams, B. A. 1989, ApJ, 339, 772

Rood, H. J., \& Struble, M. F. 1994, PASP, 106, 416

Schlegel, D. J., Finkbeiner, D. P., \& Davis, M. 1998, ApJ, 500, 525

Schombert, J. M., \& Bothun, G. D. 1987, AJ, 92, 60

Shakhbazian, R. K. 1973, Astrofizika, 9, 495

Shakhbazian, R. K., \& Petrosian, M. B. 1974, Astrofizika, 10, 13

Stoll, D., Tiersch, H., \& Braun, M. 1996, Astron. Nachr., 317, 239

Sulentic, J. W. 1987, ApJ, 322, 605

Tiersch, H., Tovmassian, H. M., Stoll, D., et al. 2002, A\&A, 392, 33 (Paper I)

Toomre, A., \& Toomre, J. 1972, ApJ, 178, 623
Tovmassian, H. M. 2001, PASP, 113, 543

Tovmassian, H. M. 2002, AN, 323, 488

Tovmassian, H. M., \& Chavushyan, V. H. 2000, AJ, 119, 1687

Tovmassian, H. M., \& Tiersch, H. 2001, A\&A, 378, 740

Tovmassian, H. M., Mazzarella, J. M., Tovmassian, G. H., Stoll, D., \& Tiersch, H. 1998, A\&AS, 130, 207

Tovmassian, H. M., Chavushyan, V. H., Verkhodanov, O. V., \& Tiersch, H. 1999, ApJ, 523, 87

Tovmassian, H. M., Yam, O., \& Tiersch, H. 2001, Rev. Mex. Astron. Astrofis., 37, 173

Tovmassian, H. M., Tiersch, H., Chavushyan, V. H., \& Tovmassian, G. H. 2003a, A\&A, 401, 463 (Paper II)

Tovmassian, H. M., Tiersch, H., Navarro, S. G., et al. 2003b, Rev. Mex. Astron. Astrofis., 39, 275 (Paper III)

Tovmassian, H. M., Tiersch, H., Navarro, S. G., et al. 2004a, A\&A, 415, 803 (Paper IV)

Tovmassian, H. M., Tiersch, H., Tovmassian, G. H., et al. 2004b, Rev. Mex. Astron. Astrofis., submitted (Paper V)

Veilleus, S., \& Osterbrock, D. E. 1987, ApJS, 63, 295

Zabludoff, A. J., Huchra, J. P., \& Geller, M. J. 1990, ApJS, 74, 1

Zepf, S. E., Whitmore, B. C., \& Levison, H. F. 1991, ApJ, 383, 524

Zickgraf, F.-J., Thiering, I., Krauter, J., et al. 1997, A\&AS, 123, 103 


\section{Online Material}


H. Tovmassian et al.: Shakhbazian compact groups. IV., Online Material p 2
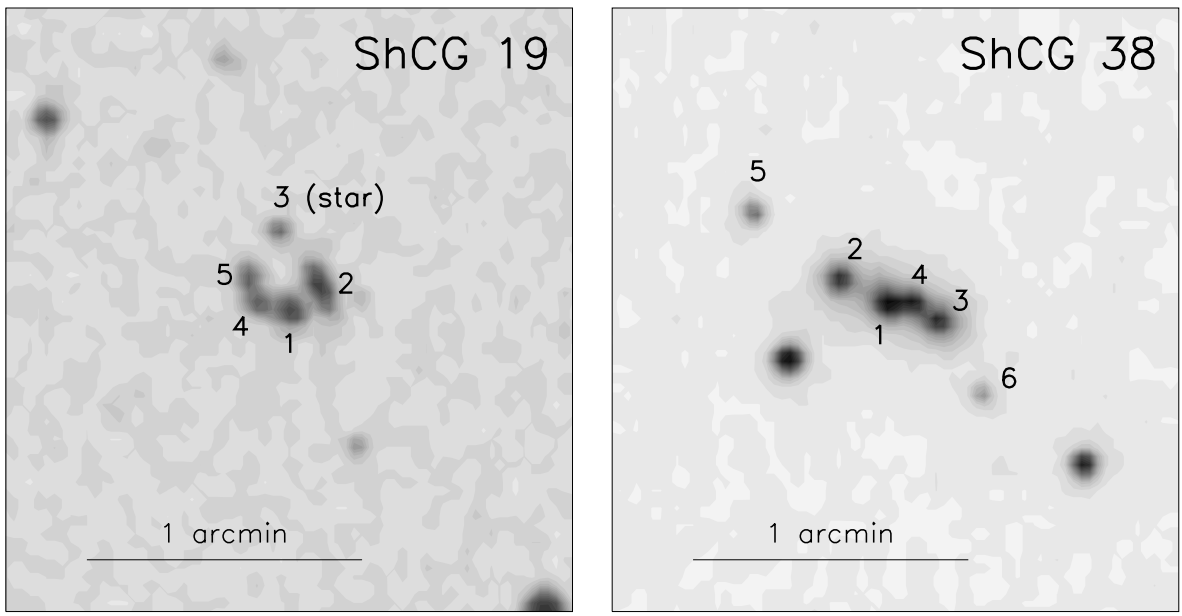

Fig. 1. The POSS red images of ShCG 19 and ShCG 38.
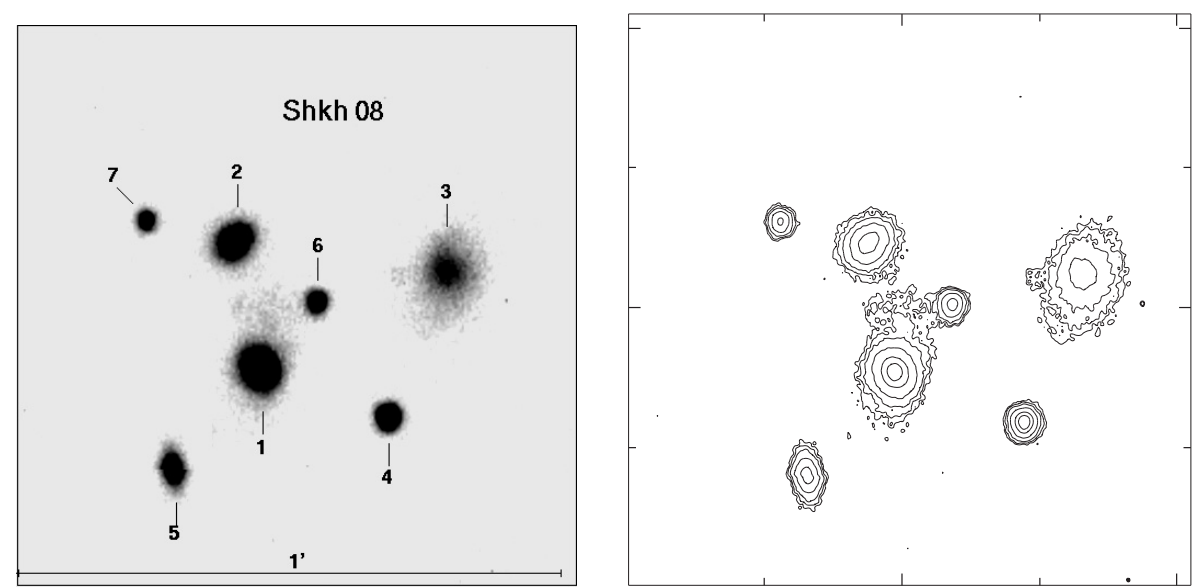

Fig. 3. The images and isophotal contourplots of the galaxies of ShCG 8 . The units of surface brightness $\mu$ of 6 isophotes starting from the innermost one are: $20^{\mathrm{m}} \cdot 22,20^{\mathrm{m}} \cdot 76,21^{\mathrm{m}} \cdot 31,21^{\mathrm{m}} \cdot 89,22^{\mathrm{m}} \cdot 76$, and $23^{\mathrm{m}} \cdot 43 . \mu=26^{\mathrm{m}} \cdot 5 / \operatorname{arcsec}^{2}$.
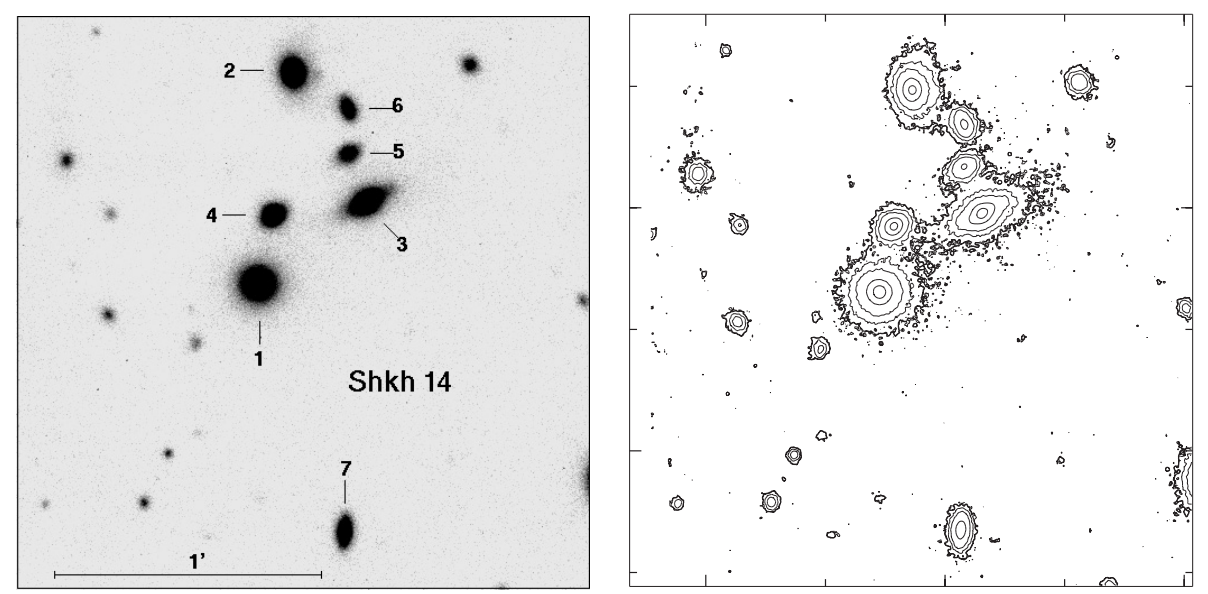

Fig. 4. The images and isophotal contourplots of the galaxies of ShCG 14. The units of surface brightness $\mu$ of 5 isophotes starting from the innermost one are: $19 . \mathrm{m} 61,20^{\mathrm{m}} 67,22^{\mathrm{m}} \cdot 70,24^{\mathrm{m}} \cdot 47$, and $27^{\mathrm{m}} .16$. 
H. Tovmassian et al.: Shakhbazian compact groups. IV., Online Material p 3
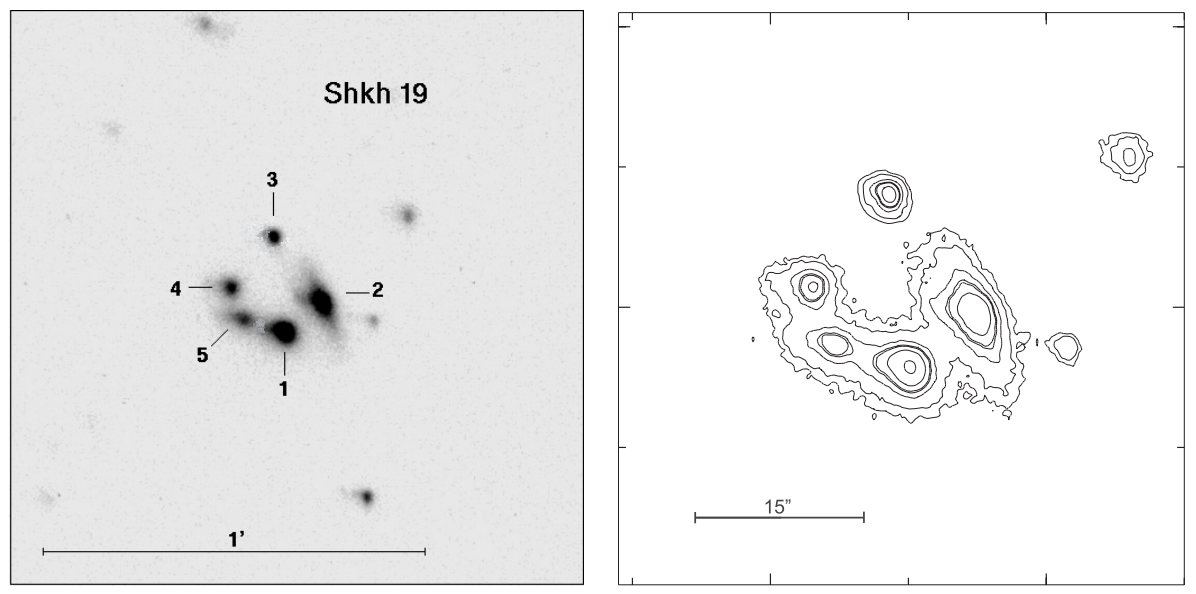

Fig. 5. The images and isophotal contourplots of the galaxies of ShCG 19. The units of surface brightness $\mu$ of 6 isophotes starting from the innermost one are: $19.07,19^{\mathrm{m}} \cdot 94,20^{\mathrm{m}} \cdot 57,20^{\mathrm{m}} \cdot 62,20^{\mathrm{m}} \cdot 92$, and $22^{\mathrm{m}} \cdot 28$.
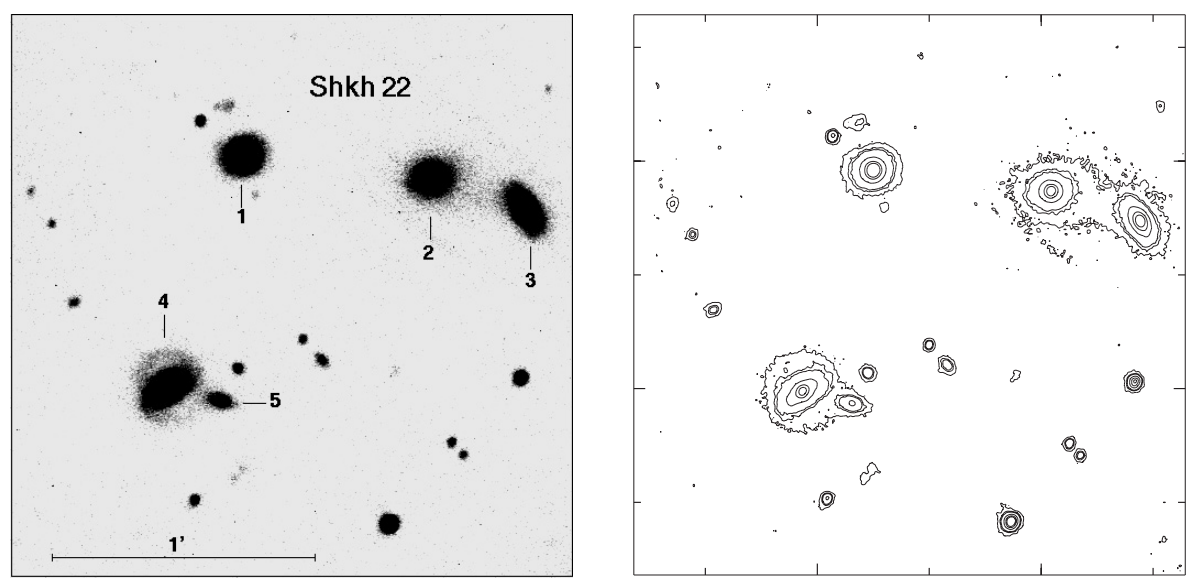

Fig. 6. The images and isophotal contourplots of the galaxies of ShCG 22. The units of surface brightness $\mu$ of 6 isophotes starting from the innermost one are: $18^{\mathrm{m}} \cdot 98,19^{\mathrm{m}} \cdot 70,20^{\mathrm{m}} \cdot 85,26^{\mathrm{m}} \cdot 55,27^{\mathrm{m}} \cdot 31,28^{\mathrm{m}} \cdot 5$.
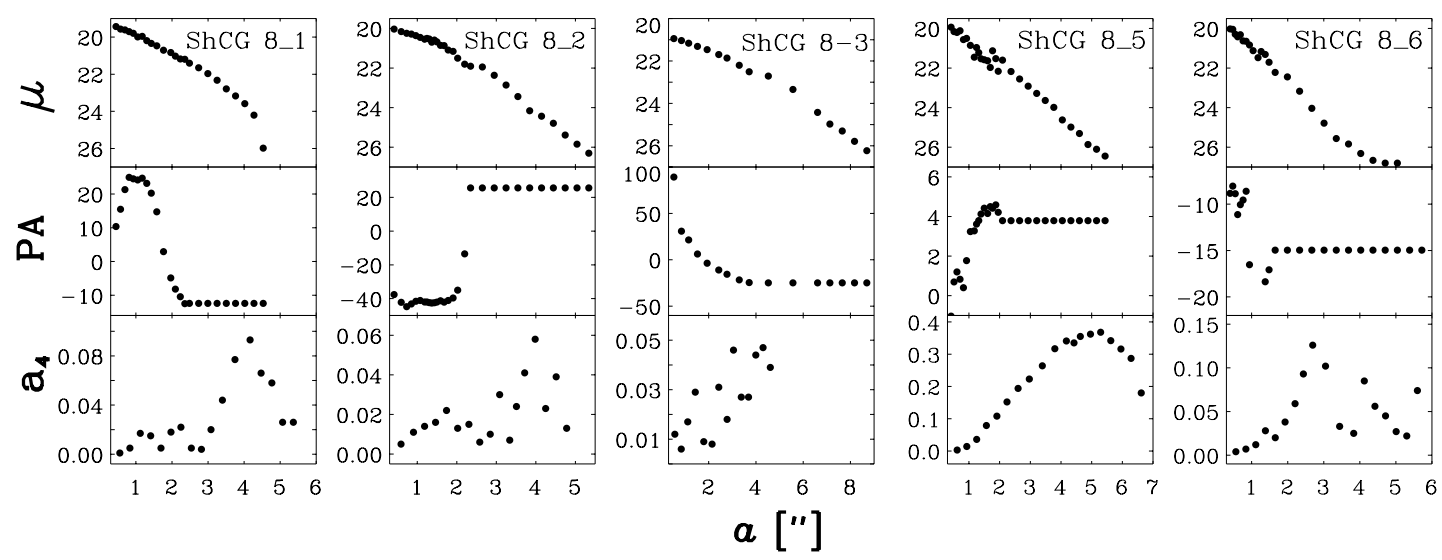

Fig. 7. The surface brightness $\mu_{R}$ the position angle PA and the Fourier coefficient $a_{4}$, versus semi-major axis $a$ of galaxies in ShCG 8 . 
H. Tovmassian et al.: Shakhbazian compact groups. IV., Online Material p 4
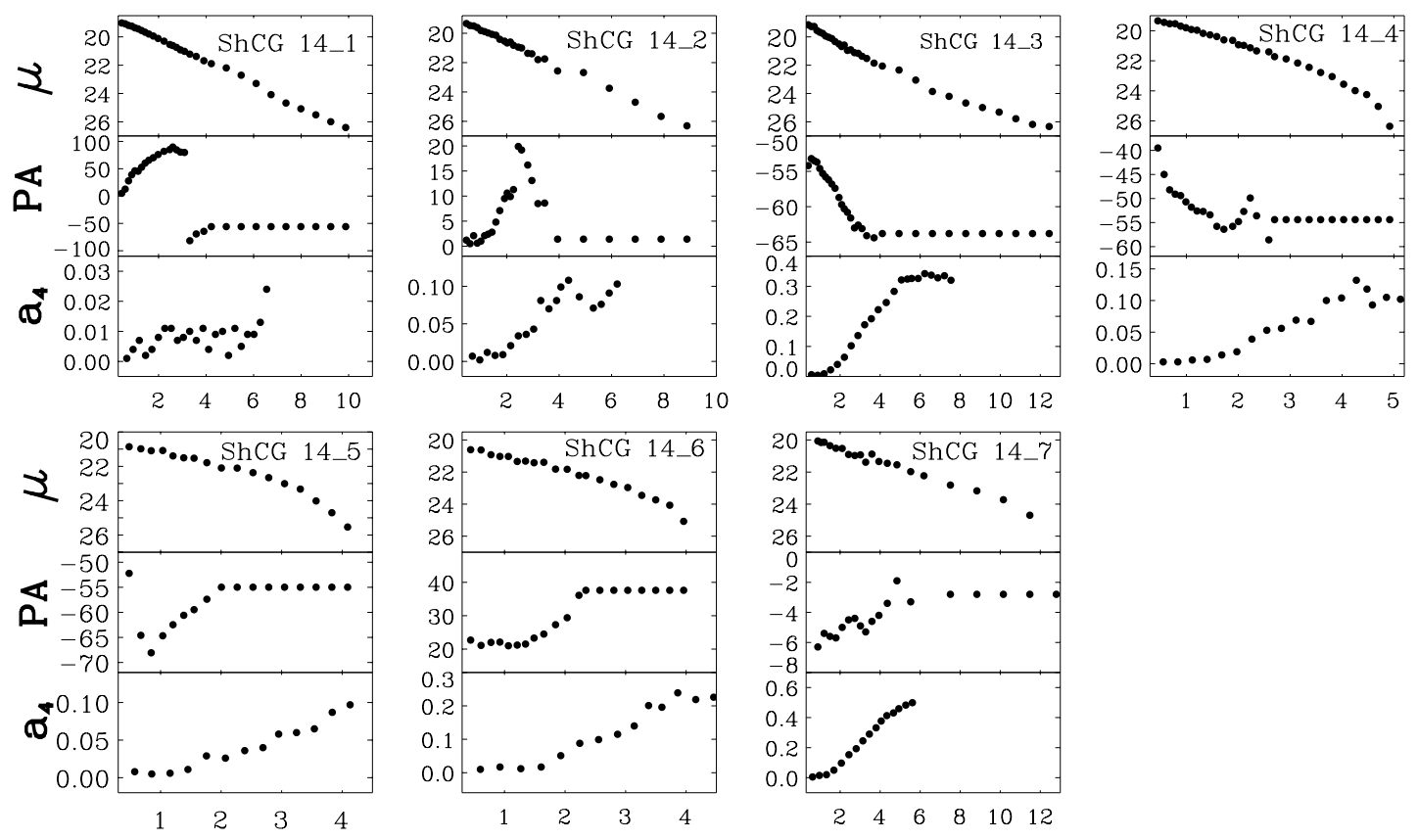

$a["]$

Fig. 8. The surface brightness $\mu_{R}$ the position angle PA and the Fourier coefficient $a_{4}$, versus semi-major axis $a$ of galaxies in ShCG 14 .
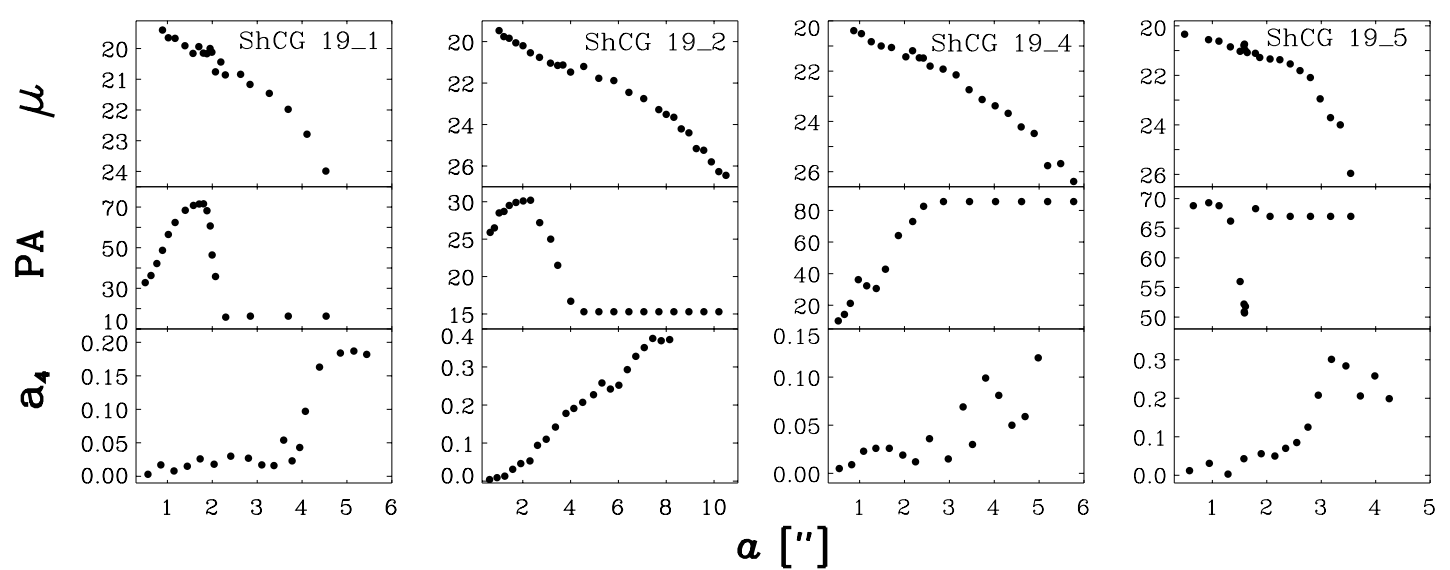

Fig. 9. The surface brightness $\mu_{R}$ the position angle PA and the Fourier coefficient $a_{4}$, versus semi-major axis $a$ of galaxies in ShCG 19 .
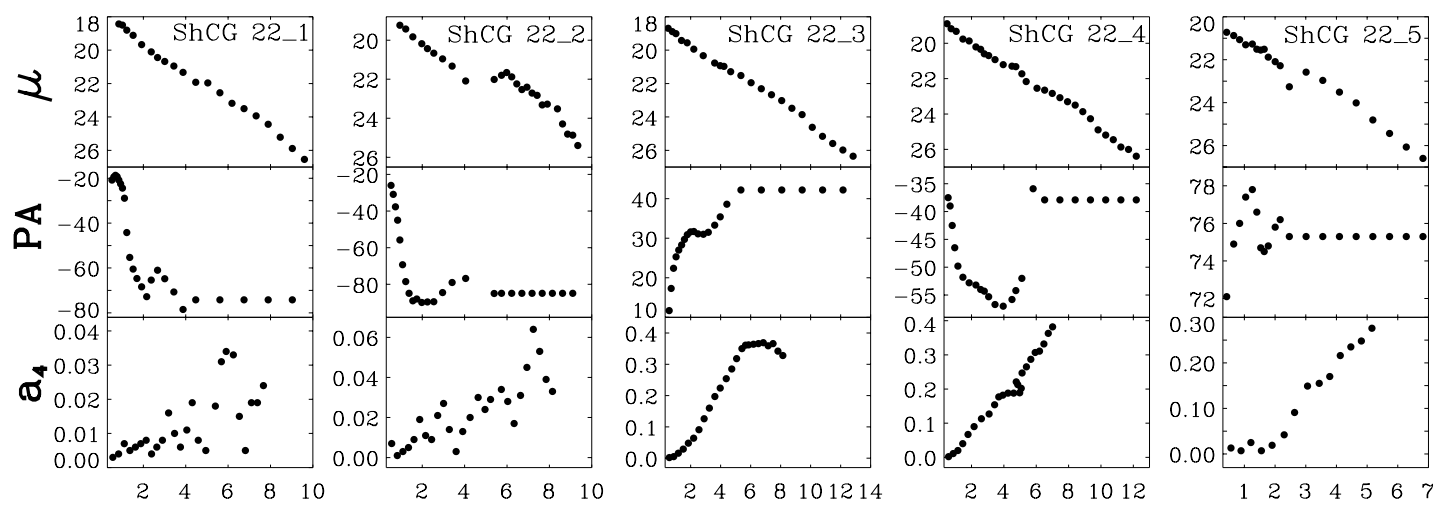

Fig. 10. The surface brightness $\mu_{R}$ the position angle PA and the Fourier coefficient $a_{4}$, versus semi-major axis $a$ of galaxies in ShCG 22 . 